\title{
COMPARISON IN THE ORAL STATUS AMONG 123 SMOKING AND NON-SMOKING PREMENOPAUSAL AND MENOPAUSAL PATIENTS
}

doi: 10.2478/rojost-2018-0052

I. Lescai ${ }^{1,4}$, D. Lescaí, M. Cîrstoiu, 314

${ }^{1}$ Blident Help Dental Clinic, Bucharest, Romania

2"Spiru Haret" University, Bucharest, Romania

3Department of Obstetrics \& Gynaecology, University Emergency Hospital, Bucharest, Romania

4"Carol Davila" University of Medicine and Pharmacy, Bucharest, Romania

A cohort of 123 patients was split into two main categories, premenopausal and menopausal. Each of the two was also split in smoking and non-smoking. The objective of the study was to find whether there is a difference between smoking and non-smoking, menopausal, and premenopausal patients. Each patient had the oral status stated on the radiological findings for missing teeth, root canal treatments and bone resorption greater than $5 \mathrm{~mm}$. In total, there were 59 premenopausal patients ( 35 non-smoking and 24 smoking) and 63 menopausal patients (39 non-smoking and 24 smoking). Non-smoking menopausal patients had 273 missing teeth, 212 root canal treatments and 163 bone resorptions greater than $5 \mathrm{~mm}$. In contrast, the smoking menopausal patients had 258 missing teeth, 70 root canal treatments, and 187 bone resorptions greater than $5 \mathrm{~mm}$. The premenopausal non-smoking patients had 128 missing teeth, 139 root canal treatments and 82 bone resorptions greater than $5 \mathrm{~mm}$. In contrast, premenopausal smoking patients had 111 missing teeth, 100 root canal treatments and 144 bone resorptions greater than $5 \mathrm{~mm}$.

As a general conclusion, premenopausal patients lost 3.65 teeth, had 3.97 root canal patients and 2.34 bone resorptions in the non-smoking group compared to 4.62 missing teeth, 4.16 root canal treatments and 6 bone resorptions for each one in the smoking group. The menopausal nonsmoking group had 7 missing teeth, 5.43 root canal treatments and 4.17 bone resorptions for every patient compared to 10.75 missing teeth, 2.91 root canal treatments and 7.79 bone resorptions for every patient in the menopausal smoking group.

Keywords: premenopause, menopause, smoking, radiography, oral status 